\title{
Tuberculous Dilated Cardiomyopathy with Myocarditis
}

\author{
Pawan Kumar $^{1}$ - Sudha Sharma ${ }^{2}$ (D) $\cdot$ Dinesh Bisht ${ }^{1} \cdot$ Rakesh Panwar $^{2}$
}

Received: 29 July 2019 / Accepted: 9 September 2019 / Published online: 24 October 2019

(C) Dr. K C Chaudhuri Foundation 2019

To the Editor: Tuberculous involvement of heart occurs in 1$2 \%$ cases and involvement of myocardium is rarer [1]. Antemortem diagnosis is delayed due to its low incidence and late diagnosis. Most of the cases occur in young age in immunocompetent males [2].

We report a rare presentation of tuberculosis in a 5-y-old girl child, who presented with fever and anasarca for 2 mo, shortness of breath, abdominal distension and cough for 2 wk. The child was born at term, immunized for age and developmentally normal. She was febrile with pulse 140/ min, blood pressure $90 / 60 \mathrm{mmHg}$, respiratory rate $38 / \mathrm{min}$ and $\mathrm{SPO}_{2} 90 \%$. Examination revealed generalised lymphadenopathy, bilateral basal crepts, muffled S1, S2, ascites and massive hepatomegaly. Chest radiograph showed enlarged cardiothoracic ratio with interstitial edema. Fine needle aspiration cytology (FNAC) of cervical lymph node showed necrosis and Zeihl Neelson stain was positive. A diagnosis of tuberculosis was given and child started on DOTS category I. Echocardiography showed dilated cardiomyopathy, moderate left ventricular dysfunction with ejection fraction $30 \%$, mild pericardial effusion and Congestive heart failure- New York Heart Association Classification (CHF-NYHA) Grade III. Creatine kinase (CK-MB) was raised (49 IU), so a final diagnosis of cardiomyopathy with tubercular myocarditis was made. Child improved on anti-tubercular treatment (ATT) and decongestive measures.

The incidence of tuberculosis is declining worldwide, however, it is still one of the most prevalent infectious etiologies. Tuberculosis usually spares the heart. The proposed

Sudha Sharma

dr.sudhasharma28@gmail.com

1 Department of Pediatrics, Dr YSPGMC, Nahan, Himachal Pradesh, India

2 Department of Pathology, Dr YSPGMC, Nahan, Himachal Pradesh, India mechanisms of cardiac involvement are hematogenous or lymphatic spread or involvement from adjacent structures [3].

Myocardial involvement, if not diagnosed early, is associated with a high mortality rate upto $30 \%[1,4]$. There are reports of postmortem diagnosis of tubercular cardiomyopathy, with relatively few cases reported antemortem [5]. This is due to its low incidence, insidious onset and progression, leading to late diagnosis [2]. In a clinically suspected case, when the diagnosis of tuberculosis is confirmed, endocardial biopsy may not be necessary [5].

There is a need to be aware of such unusual presentations to prevent misdiagnosis or delay, which may prove fatal. Early treatment is important in these cases. Appropriate investigations should be done in these cases before considering endocardial biopsy.

\section{Compliance with Ethical Standards}

Conflict of Interest None.

\section{References}

1. Agarwal R, Malhotra P, Awasthi A, Kakkar N, Gupta D. Tuberculous dilated cardiomyopathy: an under-recognized entity? BMC Infect Dis. 2005;5:29.

2. Michira BN, Alkizim FO, Matheka DM. Patterns and clinical manifestations of tuberculous myocarditis: a systematic review of cases. Pan Afr Med J. 2015;21:118.

3. Barman B, Mishra A, Ete T, et al. Tuberculosis and dilated cardiomyopathy - case report of a rare entity with literature review. Am J Med Case Rep. 2015;3:49-52.

4. Vaideeswar P, Pandit SP, Deshpande JR. Tuberculoma of the heart. Cardiovasc Pathol. 2006;15:55-6.

5. Al-Jahdali F, Al-Harbi A, Baharoon S, Al-Gamdi M, Al-Jahdali H. Tuberculous myocarditis is not always fatal: report of three confirmed cases with uneventful outcome. Int J Mycobacteriol. 2017;6:111-5.

Publisher's Note Springer Nature remains neutral with regard to jurisdictional claims in published maps and institutional affiliations. 